\title{
Solar Cycle Variation of Large-Scale Coronal Structures
}

by

Ester Antonucci and Thomas L. Duvall

April 1974

Reproduction in wole or in part

is permitted for any purpose of

the United States Government.

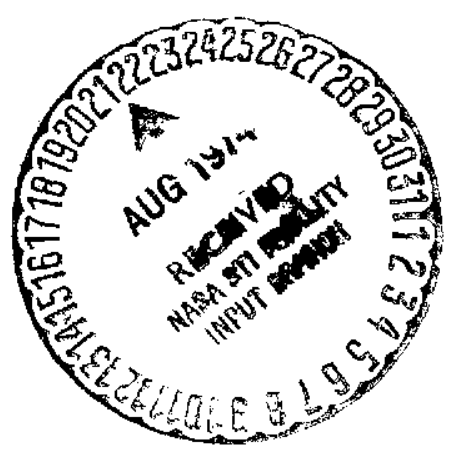

SUIPR Report No. 569

Office of Naval Research

Contract NOO014-67-A-0112-0068

National Aeronautics and Space Administration

Grant NGR 05-020-559

and

National Science Foundation

Grant GA-3!I38

(NASA-CR-139500): SOLAR CYCLE VARIATION

N74-31286

OF LABGE-SCALE CORONAL STRUCTURES

(Stanford Unit.) 19 p HC $\$ 4.00 \cdot \mathrm{CSCL} 0.3 \mathrm{~B}$ 


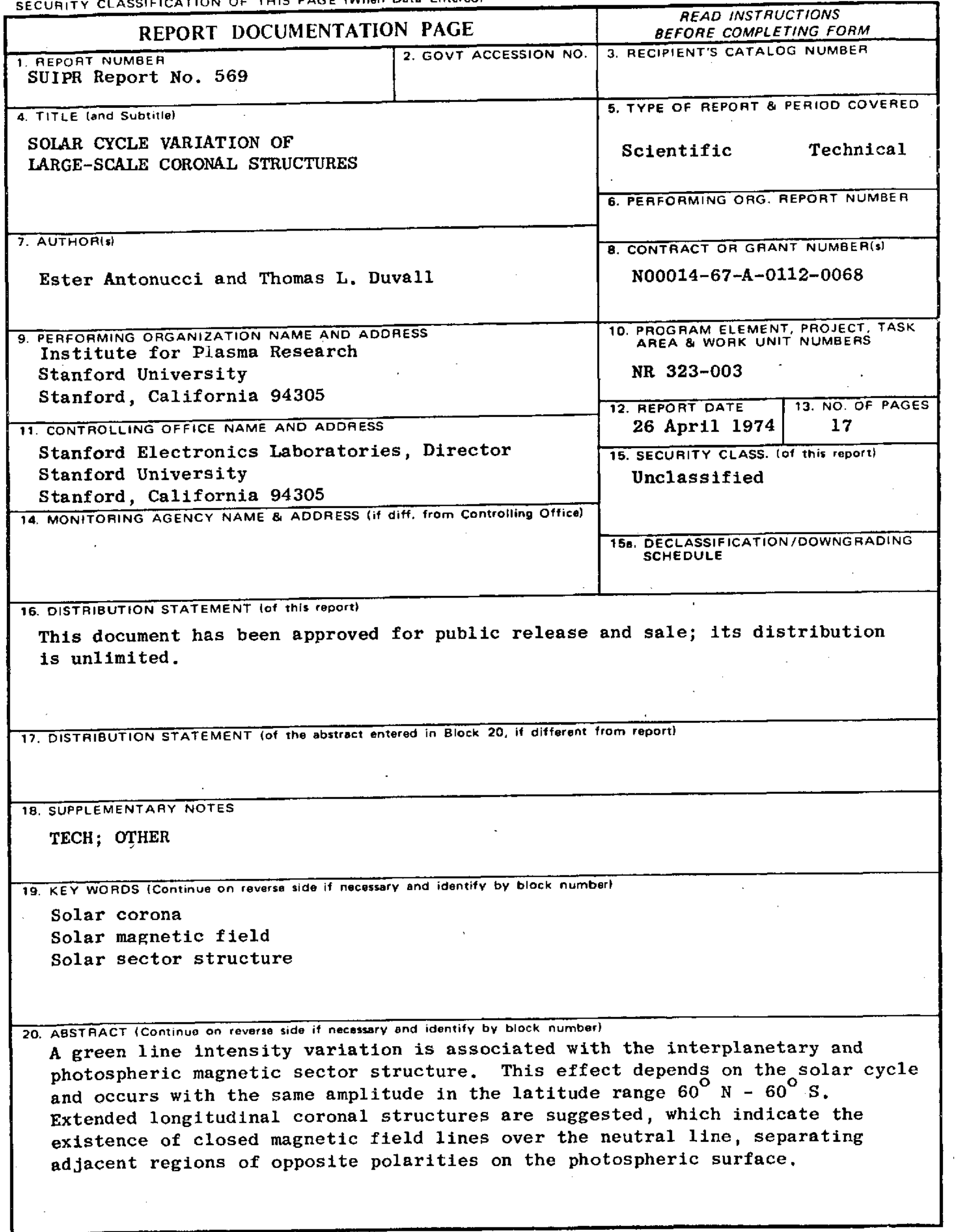


SOLAR CYCLE VARIATION OF LARGE-SCALE CORONAL STRUCTURES

by

Ester Antonucci ${ }^{*}$ and Thomas L. Duvall

Office of Naval Research Contract N00014-67-A-0112-0068

National Aeronautics and Space Administration Grant NGR 05-020-559

and

National Science Foundation

Grant GA-31138

Reproduction in whole or in part is permitted for any purpose of the

United States Government

SUIPR Report No. 569

April 1974

Institute for Plasma Research

Stanford University

Stanford, California

Submitted to Solar Physics.

* ESRO/NASA Fellow.

Now at: Istituto di Fisica - Universita di Torino - Italy. 


\title{
Solar Cycle Variation of Large-Scale Coronal Structures
}

\author{
Ester Antonucci ${ }^{*}$ and Thomas L. Duval1 \\ Institute for Plasma Research, Stanford University \\ Stanford, California 94305
}

\begin{abstract}
A green 1 ine intensity variation is associated with the interplanetary and photospheric magnetic sector structure. This effect depends on the solar cycle and occurs with the same amplitude in the latitude range $60^{\circ} \mathrm{N}-60^{\circ} \mathrm{s}$. Extended longitudinal coronal structures are suggested, which indicate the existence of closedmagnetic field lines over the neutral line, separating adjacent regions of opposite polarities on the photospheric surface.
\end{abstract}

* supported by an ESRO/NASA Fellowship. Now at: Istituto di Fisica - Universita di Torino - Italy. 
Solar Cycle Variation of Large-Scale Coronal Structures

The density structure of the corona on the large-scale is probably determined by the geometry of coronal magnetic fields. High density regions in the lower corona are usually associated with regions of strong photospheric magnetic field, But probably, more than the strength, the geometry of the field is important. Closedmagnetic configurations can be the source of enhancements in coronal density, even over weak photospheric fields. In fact the magnetic field might modulate the energy transport processes in the following way: coronal expansion and thermal conduction take place along open magnetic field lines, with consequent energy losses, which are not suffered by regions contained in closed field lines (Pneuman, 1972). Therefore the magnetic configuration of the corona might strongly influence the distribution of temperature and even more the distribution of density, because of the different energy balance mechanisms operating in regions of closed and open field lines.

At the present the information about coronal magnetic fields depends almost exclusively on their computation from the magnetic field observed on the photospheric surface. Nevertheless, there is a good agreement, at least in first approximation, between the computed coronal fields and the shapes of bright coronal emission regions. Coronal structures, such as loops and arches, are associated with an unusual high density of the coronal material.

It is important to point out that large-scale, weak photospheric fields are most influential in shaping the coronal magnetic fields. Coronal streamers, which are the broadest features of the corona, are 
commonly accepted to develop above magnetic coronal arcades, which connect large-scale unipolar regions of opposite polarities (Newkirk, 1972). Therefore adjacent large-scale photospheric regions of opposite magnetic polarities can support large-scale closedmagnetic configurations in the corona, which may play an important role in the density distribution of the coronal gas and consequently on the brightness distribution.

The relationship between coronal enhancements and large-scale photospheric fields is also suggested by the fact that coronal bright regions rotate with the large-scale magnetic structures on the surface, rather than with active regions (Wilcox and Howard, 1970; Antonucci and Svalgaard, 1974a).

The spacial association in the corona of high magnetic arcades and streamers confirms the picture of the helmet streamer as consisting of a region of closed magnetic loops (helmet), with open field lines adjacent to and above the loops. The open field lines extend far away in the interplanetary space and give origin to a current sheet, which separates the two regions of opposite polarities of the streamer. These neutral sheets constitute sector boundaries in the interplanetary magnetic field. Therefore the large-scale unipolar regions of the photosphere, which originate an interplanetary sector boundary, are likely to be the foot of closedfield lines (closedmagnetic region of a streamer) up to about

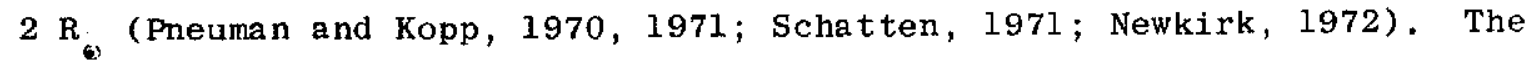
coronal gas is confined magnetostatically in the closedmagnetic loops, with an increase in temperature and density (Pneuman, 1972). Even if every high magnetic arcade system might form a coronal streamer with the associated current sheet, only the systems with axes lying near a meridian can 
produce a sector boundary in the interplanetary field at $1 \mathrm{~A} . \mathrm{U}$. In this case they are unlikely to be seen as coronal streamers at the $1 \mathrm{imb}$, as in the case of arcade systems with horizontal axes (Newkirk, 1972). A clear example of association of computed magnetic arcades in the corona and interplanetary sector structure has been discussed by Wilcox and Svalgaard (1974).

Interplanetary magnetic sector boundaries detected at 1 A.U. can be therefore interpreted as manifestations of current sheets connected with coronal streamers, which appear essentially as closed loops inside $2 \mathrm{R}_{\odot}$. This suggests that interplanetary magnetic sector boundaries should be related to enhancements in the coronal brightness, due to coronal condensations in regions of closedmagnetic configuration. This relationship should hold at least for the coronal brightness at low heliolatitudes.

Associations of coronal enhanced features and interplanetary sector boundaries have been indeed observed. Martres et al (1970) suggest that interplanetary sector boundary, traced back to the solar surface, originates $14^{\circ} \mathrm{W}$ of a coronal condensation, from an analysis of radio observations. A peak of solar radio emission, preceding the inferred central meridian passage of the interplanetary sector boundary, has been found by Scherrer and El-Raey (1974) for the period 1962-1970. Also the green line intensity seems to be affected by the large-scale solar magnetism. Guldbrasen (1973) notes that during the period 1962-64 the green line emission peaks in a region west of the solar sector boundaries. The association of photospheric sector boundaries, as inferred from the interplanetary magnetic field polarity data, with coronal enhance- 
ments is more complicated than might appear from the previous analyses. In fact from an analysis of the green line intensity (Antonucci, 1974) it seems to depend both on the kind of sector boundary considered, separating $(+,-)$ or $(-,+)$ polarities, and on the solar hemisphere. Moreover the relationship of coronal features and magnetic sector boundaries displays a solar cycle variation. During a given sunspot cycle, green line intensity maxima are closely related to the solar magnetic boundaries, which separate polarities coinciding with the leading and following polarities of bipolar sunspot groups of the solar hemisphere considered. Therefore when a $(+,-)$ sector boundary in the northern hemjisphere is associated with enhanced green coronal emission, in the southern hemisphere a (-,+) sector boundary is near a coronal condensation. Because this effect is independent of latitude from $0^{\circ}$ up to $60^{\circ}$ in latitude, persistent northern and southern coronal features, extending up to high latitudes, should exist. Their relationship with the magnetic sector structure implies a longitudinal separation of northern and southern features at least at high latitudes, of roughly $90^{\circ}$ or $180^{\circ}$, respectively in the case of a 4-sector or 2-sector structure. This hypothesis has been verified by Antonucci and Svalgaard (1974b).

A solar cycle dependence of the variation of the green line intensity, in the frame of the magnetic sector structure, (Antonucci, 1974) has been found by means of cross-correlations of green line intensity data and Interplanetary polarity data for the period 1947 - 1970 . The green line Intensity data have been prepared by sykora (1973) in the form of synoptic tables. The Pic du Midi photometric scale has been used to normalize the intensity data. The observations 
are referred to central meridian by averaging the intensities measured at the limbs seven days before and seven days after. The original tables report the data as three-day averages for six latitude zones, $20^{\circ}$ wide, from $57^{\circ} .5$ South to 57.5 North. In order to compare these data with the daily interplanetary polarity data, daily values have been interpolated from sykora's tables. Moreover a 27-day running mean has been subtracted from the green line intensity data, in order to remove long-term variations of the coronal emission, related to the sunspot cycle. The polarity data of the interplanetary megnetir field are inferred from high latitude geomagnetic observations (Svalgaard, 1972). They can be expressed in a time-series of +1 and -1 daily values, respectively for days in which the polarity of the interplanetary magnetic field is positive (away from the Sun) or negative (toward the Sun).

The cross-correlations between green corona emission and interplanetary magnetic field polarity have been performed for consecutive periods, two-years long, for each latitude zone. From the interpretation of the cross-correlation functions the time-lag between the center of the positive interplanetary magnetic sector and the coronal enhancement was deduced for each latitude and period, through the interval 1947 - 1970. Then, assuming the size of the sector of roughly.7 days, in the hypothesis of a 4-sector structure, the relative time-lag between sector boundary and coronal enhancement was inferred. But the size of interplanetary sectors is slowly changing, moreover, during the active part of a sunspot cycle, the sector structure of the interplanetary ma - $^{-}$ netic field consists of two sectors (Wilcox et al, 1972). Therefore the 
cross-correlation analysis, discussed before, gives correct results for the individuation of the kind of boundaries which are associated with coronal enhancements and the solar cycle dependence of the effect. But this method is not ideal for a very precise determination of the temporal and spacial relationship between magnetic boundaries and coronal intensity. An improved and more detailed analysis can be done if the coronal variations associated with the magnetic sector structure are directly computed, in the frame of the sector boundaries; provided that the two kinds of sector boundaries are analyzed separately. This kind of study confirms all the characteristics of the effect deduced from the cross-correlation analysis and at the same time gives a clearer picture of the effect at the different latitudes.

Superposed epoch analyses of the green line intensity around the interplanetary sector boundaries have been performed for consecutive periods, two-years long, centered in each year of the interval $1948-1969$. The days in which the derivative of the polarity data time series is different from zero have been selected as sector boundaries of the interplanetary magnetic field. The polarity data have been previously corrected in order to avoid contributions from short-term polarity changes to the number of sector boundaries, $i . e$. we have reversed the polarity of each day followed and preceded by two consecutive days of polarity opposite to that day. The two kinds of sector boundaries, separating $(+,-)$ or $(-,+)$ polarities, are distinguishable by the sign of the derivative, indicating the change of magnetic polarity. 
In Figure 1 the superposed epoch analyses relative to the year 1952 (from the middle of 1951 to the middle of 1953) are reported for each latitude zone, respectively for the $(+,-)$ and the $(-,+)$ sector boundary. The zero day is the time of the interplanetary sector boundary at 1 A. U. The vertical lines mark the day of the solar sector boundaries, at central meridian approximately 4.5 days before the interplanetary boundaries are detected, in order to account for the sun-earth transit time (Wilcox et al., 1969). For the period considered, $36(+,-)$ boundaries and $33(-,+)$ boundaries are taken into account in computing the superposed epoch analyses of the green line intensity.

Each plot of Figure 1 represents the average variation in percentage of the green line intensity associated with a $(+,-)$ or $(-,+)$ sector boundary, at each latitude zone. The zero level of the variation" is due to the fact that a 27 -day running mean has been subtracted first from the intensity data, in order to remove the solar cycle modulation, as explained earlier. The uncertainty of the average coronal intensity, around the boundary, has been computed by dividing the standard deviation by the square root of one less than the number of boundaries used. The error bars, reported in Figure 1 , represent twice the uncertainty computed for the maximum or minimum value of the average intensity in each plot.

The variations of the green line intensity, in the frame of the solar magnetic sector structure, are statistically significant. Their amplitudes, of the order of $10 \%$ or more, are independent of latitude in the range considered $57^{\circ} .5 \mathrm{~N}-57^{\circ} 5 \mathrm{~S}$. Therefore, the percentage of 
coronal emission involved in this effect is approximately the same at each he1iolatitude.

For a given latitude, one kind of boundary is associated with an enhancement in coronal emission, while the other kind is associated with a dip in emission. Morecver in the northern hemisphere (top half of Figure 1) the emission peak is assoclated with the $\left(-b_{+}\right)$boundary, while in the southern hemisphere (bottom part of Figure 1) it is associated with the $(+,-)$ boundary.

The information provided by the 22 sets of superposed epoch analyses (analogous to the set shown in Figure 1) relative to the whole period 1948 - 1969, can be represented in a rather simple way. For each intensity variation, for a given latitude and a given boundary, we consider just the days in which the average intensity differs from the zexo level more than the uncertainty. Such selected days correspond to the maxima and minima of the intensity variations. In Figure 2 , for each year of the interval 1948 - 1969, the maxima (dark areas) and minima (dashed areas) of the coronal emission have been plotted in the interval \pm 3 days around the time of the inferred sector boundaries at centra1 meridian, for the low latitudes of the northern hemisphere $\left(17^{\circ} .5 \mathrm{~N}-2.5 \mathrm{~S}\right)$. The two kinds of sector boundaries are represented separately. The horizontal dashed lines separate one solar cycle from the other.

Even a quick look at Figure 2 can convince that the effect of a coronal intensity variation with maximum and minimum at different kinds of boundaries is confirmed for the whole period considered, with very few exceptions. Another interesting aspect of the coronal modulation is evident immediately: the pattern of the green line intensity variation reverses 
with respect to the solar magnetic sector structure at each sunspot minimum.

The solar cycle variation of the coronal modulation suggests a correspondence between the polarities of leading and following sectors, which form a boundary close to a coronal enhancements, and the leading and following polarities of sunspot groups. In fact Figure 2 shows that in the northern hemisphere the boundary associated with coronal enhancements is separating $(-,+),(+,-)$ and again $(-,+)$ polarities, respectively in cycle 18, 19 and 20 . For each solar cycle, the boundary polarities are in the right sequence representing the preceding and following polarities of sunspot groups in the northern hemisphere.

This correspondence in polarity can still be found analyzing the latitudinal dependence of the solar cycle variation of the effect that we are studying. In Figure 3 for each latitude the maxima and minima of the coronal intensity variations are reported. Only the $(+,-)$ sector boundary is considered, because the $(-,+)$ boundary displays simply the reversed situation of maxima and minima, as seen in Figure 2.

The first three plots refer to the northern hemisphere and indicate clearly the same patterns. Therefore from the equator up to $57^{\circ} .5 \mathrm{~N}$, the solar cycle dependence of the coronal modulation is the same as we have discussed for the low latitudes represented in Figure 2. The first part of cycle 20 is anomalous at high latitudes $\left(57^{\circ} .5-37^{\circ} .5 \mathrm{~N}\right)$.

The last three plots refer to the southern hemisphere. For this hemisphere, the green line intensity variations are irregular at low latitudes. In fact, for this latitude zone, cycles 19 and 20 show patterns 
that resemble the northern ones more than the southern ones, when the variations of the green line intensity are significant. However high and middle latitudes $\left(17^{\circ} .5-57^{\circ} .5 \mathrm{~S}\right)$ show the same solar cycle variation of coronal emission.

Comparing the two hemispheres, in the cycles in which minima of intensity are present at the $(4,-)$ boundary at North, at South we find coronal enhancements and vice versa. This reversal of the pattern of green Iine intensity maxima and minima with the hemisphere is consistent with the suggestion that the boundary associated with coronal enhancements separates polarities corresponding to the sunspot group polarities (in the East-West sequence). Preceding and following polarities of sunspot groups are $(+,-)$ in the southern hemisphere during cycle 18 and 20 , and in the northern hemisphere during cycle 19 . The $(+,-)$ sector boundary is associated with peaks in coronal emission, in the southern hemisphere during cycle 18 and 20 , and in the northern hemisphere during cycle 19 , as shown in Figure 3 .

The butterfly diagram for sunspots (Stenflo, 1972) indicates that, during solar cycle 20, sunspots show up at the beginning of 1965 in the northern hemisphere, while in the southern hemisphere their appearance is delayed until the beginning of 1966. In Figure 3 the dashed 1 ine, separating cycles 19 and 20 at the beginning of 1965 , marks the reversal of the effect, expected at sunspot minimum, only at northern latitudes. At South the reversal takes place one year later in 1966 , at middle and high latitudes, in coincidence with the appearance of sunspots in that hemisphere. 
Another peculiarity occurs in 1961. At this time a disappearance or reversal of the effect takes place at every latitude. Stenflo (1972) shows, in a butterfly diagram of the photospheric magnetic field detected at Mt. Wilson, that a unique abrupt reversal of the photospheric field occurs in the same year at least at middle and low latitudes.

In conclusion this analysis of the green coronal emission, in regions close to magnetic sector boundaries, clearly indicates that the large-scale solar magnetism modulates the coronal brightness distribution. This modulation is solar cycle dependent in a way which suggests a link between large-scale and sunspot magnetism, because of the correspondence of polarities of magnetic boundaries and sunspot groups in a given hemisphere.

Moreover, because the green line intensity variations in a given hemisphere are present up to $60^{\circ}$ latitude, we suggest longitudinal northern and southern coronal structures, corotating with the large-scale magnetic pattern and not taking part in differential rotation. In case of differential rotation, middle and high latitudes would not present a variation with respect to an interplanetary time reference such as sector boundaries on a time-scale of two years. If southern and northern features are linked or not on the solar surface remains unclear because of the difficulty of interpreting the behaviour of low southern latitudes. However, a connection between southern and northern features cannot be excluded.

These extended coronal condensations on the solar disk are probably a manifestation of the real photospheric boundary or neutral line separating opposite polarity regions, over which closed field lines develop. 


\section{Acknowledgements}

We thank Dr. John M. Wilcox for helpful discussions.

Dr. E. Antonucci is an ESRO/NASA fellow. This work was supported in part by the office of Naval Research under contract N00014-67-A-0112-0068, by the National Aeronautics and Space Administration under Grant NGR 05-020-559, and by the Atmospheric Sciences Section of the National Science Foundation under Grant GA-31138. 


\section{$\underline{\text { References }}$}

Antonucci, E.: 1974, Solar Physics, "Eleven-Years Inversion of the Green Corona Emission", to be published.

Antonucci, E, and Svalgaard, L.: 1974a, Solar Physics, "Rigid and Differential Rotation of the Solar Corona", to be published.

Antonucc1, E. and Svalgaard, L.: 1974b, Solar Physics, "Green Corona and Solar Sector structure" to be published.

Guldbrasen, A.: 1973, Planetary Space Science, 21, 2003.

Martres, M., Pick, M. and Parks, G. K.: 1970, Solar Physics, 15, 48.

Newkirk, G., Jr.,: 1972, "Coronal Magnetic Fields and Solar Wind", Solar Wind, ed.: C. P. Sonett, P. J. Coleman, Jr. and J. M. Wilcox. NASA SP-308, Washington, D.C.

Pneuman, G. W.: 1972, Solar Physics, 28, 247.

Pneuman, G. W. and Kopp, R. A.: 1970, Solar Physics 18, 250.

Pneuman, G. W. and Kopp, R. A.: 1971, Solar Magnetic Fields, ed. R. Howard, p. 526 .

Schatten, K. H.: 1971, Cosmic Electrodynamics 2, 232 .

Scherrer, P. H. and E1-Raey, M.: 1974, Solar Physics, to be published. Sýkora, J.: 1973, Contr. Astron. Observ., Skalnate Pleso 5, 5.

Svalgaard, L.: 1972, Danish Meteorol. Inst. Geophys., Pap. 2.

Stenflo, J. O.: 1972, Solar Physics, 23, 307.

Wilcox, J. M., Severny, A. and Colburn, D.S.: 1969, Nature 224, 353.

Wilcox, J. M. and Howard, R.: 1970, Solar Physics 13, 251.

Wilcox, J. M. and Colburn, D. S.: 1972, J. Geophys. Res., 77, 751.

Wilcox, J. M. and Svalgaard, L.: 1974, Solar Physics, "Coronal Magnetic Structure at a. Solar Sector Boundary," to be published. 


\section{Figure Captions}

Figure 1. The average variations of the green line intensity relative to the period middle 1951 - middle of 1953 are plotted around the $(+,-)$ and $(-,+)$ sector boundaries of the solar magnetic field at central meridian (vertical lines). The variations are expressed in percentage; the mean values of the green line intensity for the six latitude zones, from the North to the South, are respectively $21,36,53,46,26$, 16 in absolute coronal units (millionths of energy radiated from the center of the Sun's disk in $1 \AA$ strip of the spectrum near the corona emission line).

Figure 2. Maxima (dark areas) and minima (dashed areas) of the average green line intensity variations around the $(+,-)$ and $(-,+)$ solar boundaries. The intensity variations are computed by means of superposed epoch analysis for periods two-years long, centered in each year of the internal 1948 - 1969, and for the northern low latitudes.

Figure 3. For each latitude zone, in the range $57^{\circ} .5 \mathrm{~N}-57^{\circ} .5 \mathrm{~s}$, the maxima (dark areas) and minima (dashed areas) of the green line intensity variations around the $(+,-)$ solar boundary are reported, for the interval 1948 - 1969. 
GREEN CORONAL EMISSION SUPERPOSED EPOCH ANALYSIS

WITH RESPECT TO MAGNETIC SECTOR BOUNDARIES

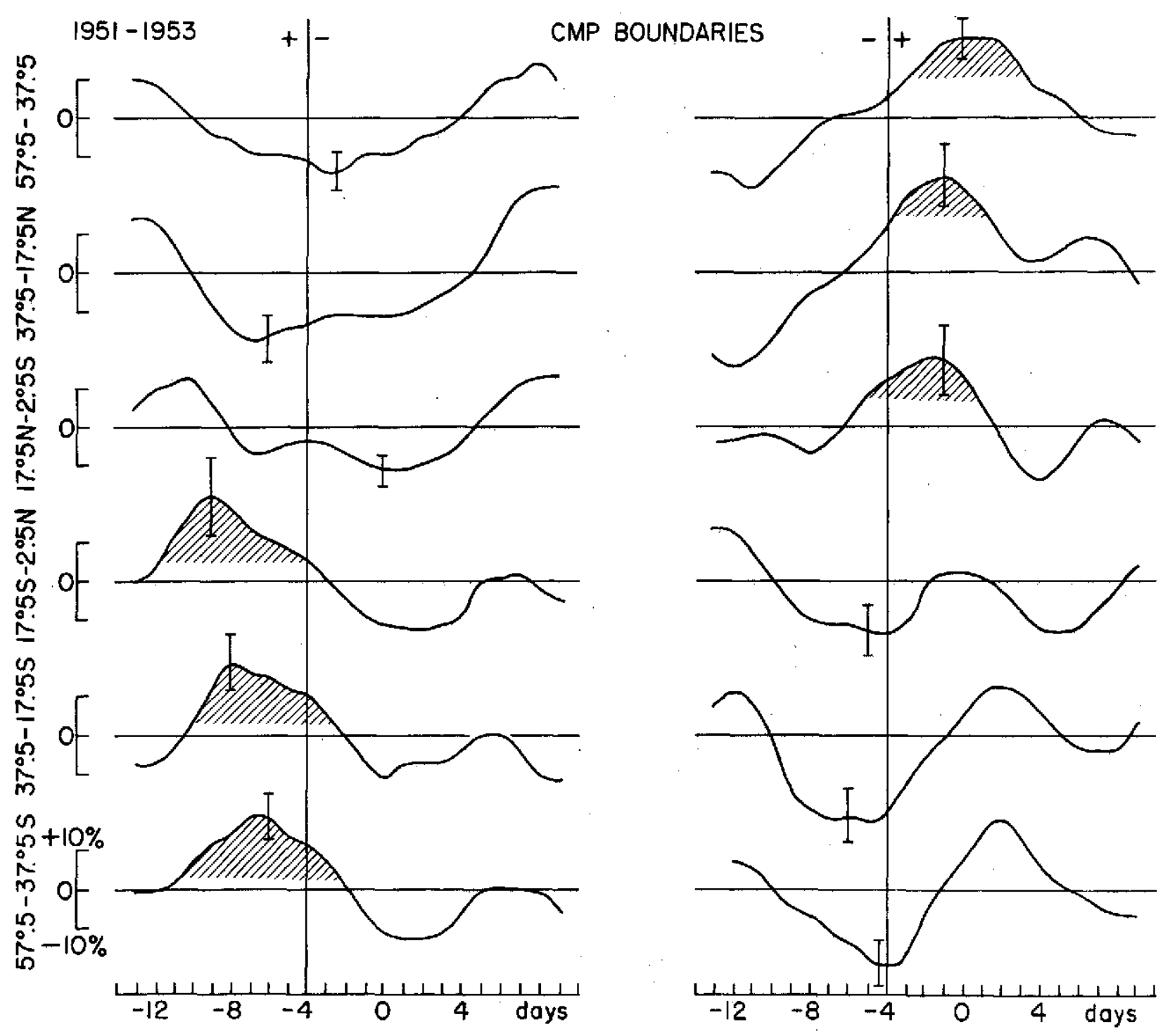

Figure 1 
GREEN CORONAL EMISSION 17:5N-2:5S AT SOLAR MAGNETIC BOUNDARIES

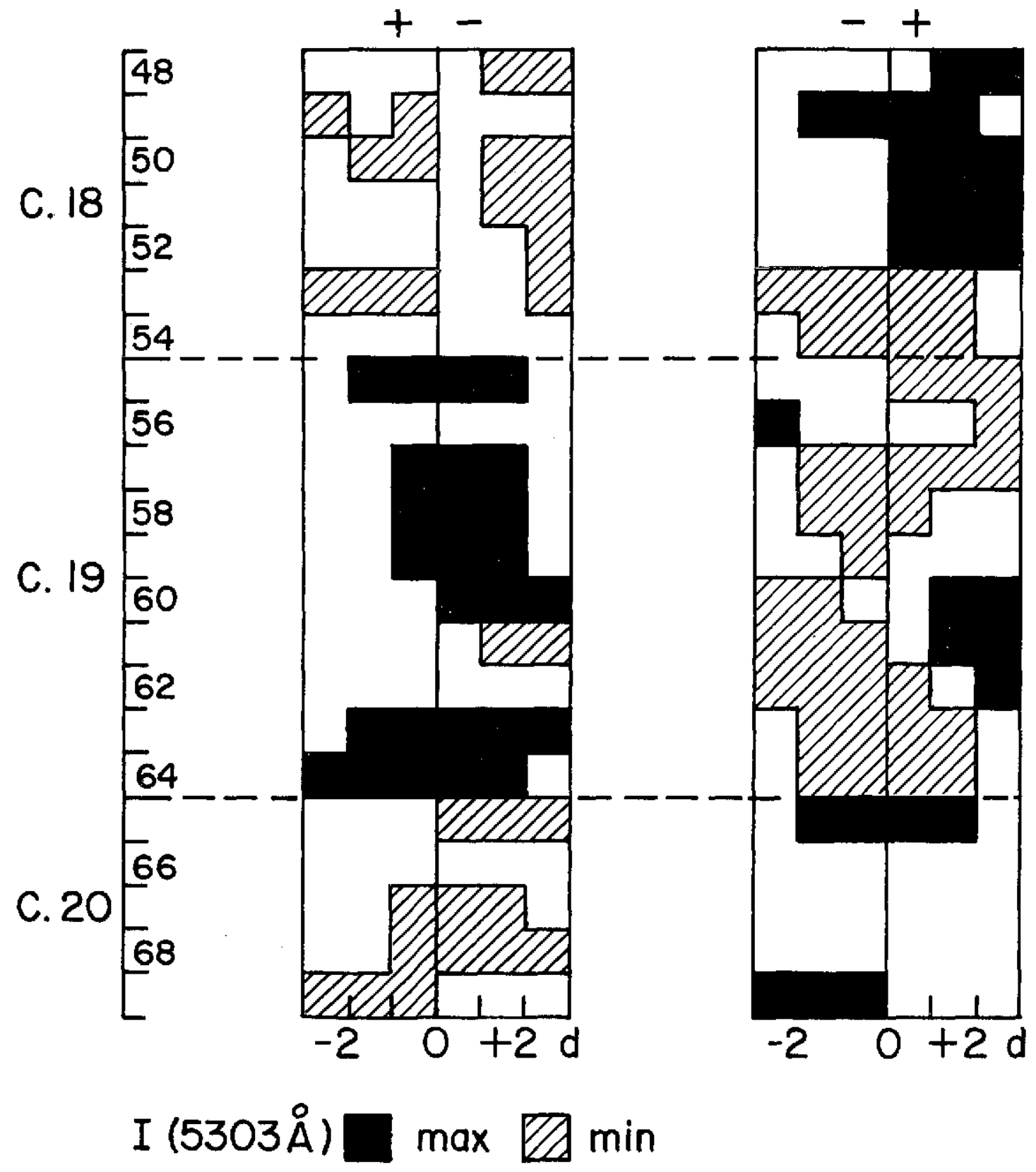

Figure 2 
GREEN LINE CORONAL EMISSION AT THE $(+,-)$ SOLAR MAGNETIC BOUNDARIES

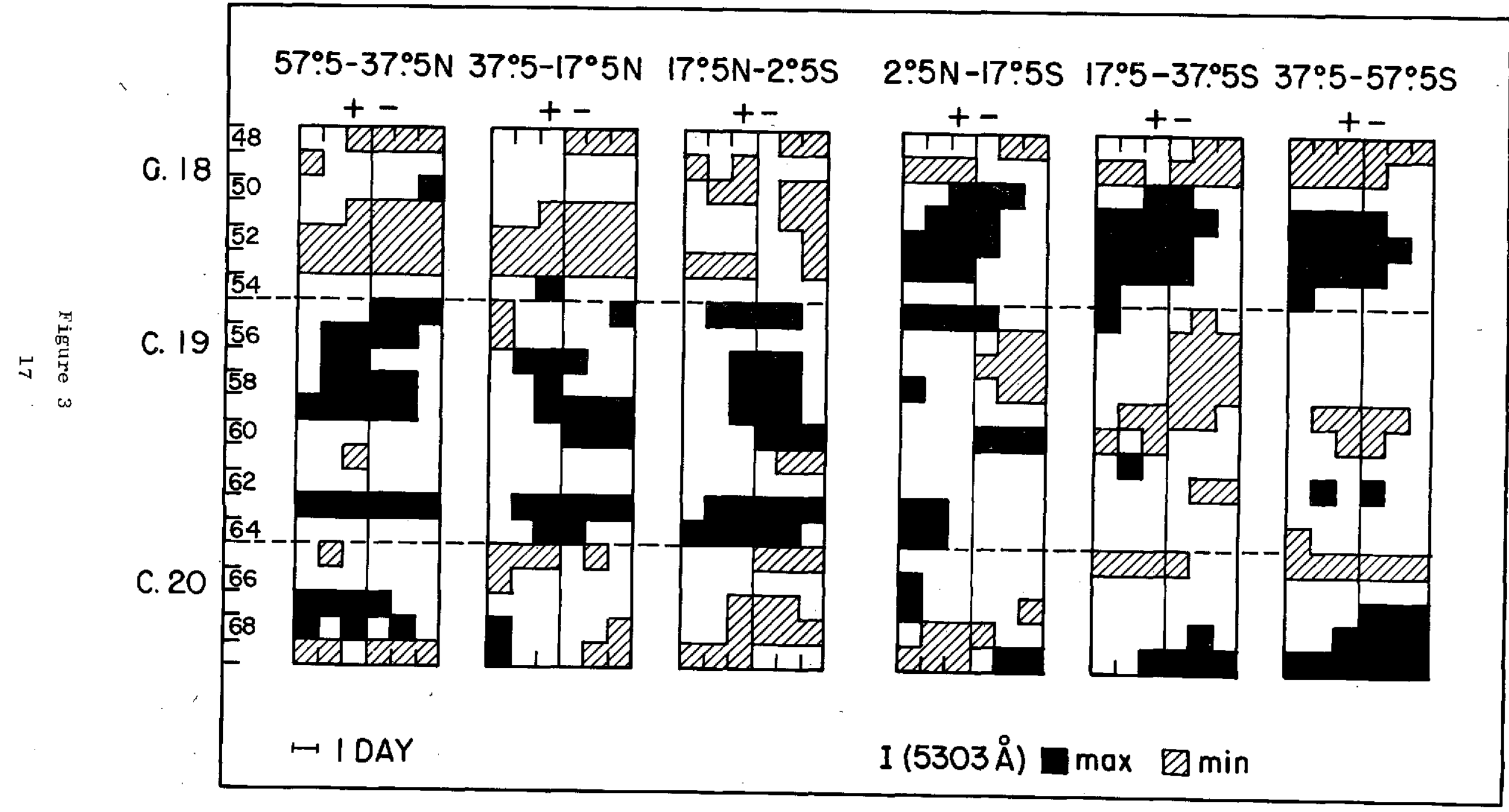

